\title{
An Investigation of X-ray Mapping/Imaging and the Artifacts Present Using a Silicon Drift Detector - Is Post-Collection Pile-Up Correction Essential?
}

\author{
B. J. Griffin ${ }^{1}$, D.C. Joy ${ }^{2}$, J. R. Michael ${ }^{3}$ and J.R. Muhling ${ }^{1}$
}

1 Centre for Microscopy, Characterization and Analysis, The University of Western Australia, Crawley, WA Australia 6009

2 Center for NanoPhase Materials Science, Oak Ridge National Laboratory, Oak Ridge, TN 37831

3 Sandia National Laboratories, PO Box 5800, Albuquerque, NM 87185-0886

$\mathrm{X}$-ray analysis and element mapping has been revolutionized by the introduction of the Silicon Drift Detector (SDD) due to its ability to collect and process the x-ray signal at very high count rates ${ }^{1}$. This study investigates the characteristics of the $\mathrm{x}$-ray data from a SDD with respect to $\mathrm{x}$-ray map resolution and $\mathrm{S}: \mathrm{N}$ in comparison to electron imaging under the same conditions.

A $30 \mathrm{~mm}^{2}$ JEOL SDD mounted on the field emission sourced JEOL $8530 \mathrm{~F}$ electron microprobe has been the main system used. A silicon wafer sample was abraded with 240 grade carborundum paper to provide a sample that had a simple composition, was homogeneous, conductive and relatively flat and with a high level of surface detail at a scale from microns to nanometers. Full spectrum mapping (10ev/channel, 2048 channel) was performed at primary electron beam energies of 30,20,10 and 5kV. Electron beam currents ranged from $\sim 5 \mathrm{nA}$ (@30 kV) to $\sim 80 \mathrm{nA}(@ 5 \mathrm{kV})$, to provide an input count rate of $\sim 65 \mathrm{k} \mathrm{cps}$ and deadtime of $\sim 46 \%$. These counting conditions were selected to give SDD operation within, but very close, to the maximum recommended processing rate. Spectral maps were collected using the frame integrating "fast scan" mode where repeated short dwell time sample scans progressively accumulate. Collection was halted when the maximum pixel value neared the 8-bit limit of 255, to match the SE imaging at a first approximation. Typically maps required $60-80$ mins to complete and contain $\sim 2 \times 10^{8}$ counts. Integrated 8-bit pixel values totals for the $\mathrm{SE}$ images are $\sim 1 \times 10^{8}$. Images and 'maps' have been processed and analysed using the NIH Image J $1.37 \mathrm{R}$ with the SMARTeR routines ${ }^{2}$. X-ray spectra have been processed using Microsoft excel.

The x-ray spectra compiled from the full spectrum x-ray maps contain a very high number of counts and consequently have very good S:N, allowing small peaks to be accurately measured and identified. The principal feature of all $\mathrm{x}$-ray spectra is the presence of sum or 'pile-up' peaks (figure 1). At high beam energy $(30 \mathrm{kV})$, these are $\mathrm{Si} \mathrm{Ka}$ interferences of first and second order, appearing at $3.78 \mathrm{keV}$ and $5.22 \mathrm{keV}$ respectively. Using a simple background subtraction gives them as $\mathrm{x} \%$ and $\mathrm{y} \%$ of the parent peak respectively. At low beam energy $(5 \mathrm{kV})$ the shallower beam penetration results in a higher contribution from surface carbon and oxygen $\mathrm{x}$-rays to the collected signal. These in turn add a complexity to the spectrum with $\mathrm{C} \mathrm{Ka}-\mathrm{Si} \mathrm{Ka}$ and $\mathrm{O} \mathrm{Ka}-\mathrm{Si}$ Ka pile-up also clearly resolved. The 'obvious' pile-up peaks distract attention from continuum pile-up with principal emission lines, the result being a significant distortion of the Bremsstrahlung with resultant errors in background corrections.

A simple channel-by channel pile-up correction has been applied, based on a routine originally written for quantitative analysis using a $\mathrm{Si}(\mathrm{Li}) \mathrm{EDS}$ in $1979^{3}$. The results confirm findings from other studies of post-collection pile-up correction for $\mathrm{SDD}^{4}$; it is essential. 
The x-ray maps have a very low S:N. To provide a value the original SMART S:N routine has been modified by adding a $\times 10^{3}$ multiplier into the final SNR calculation. The original code took the integral of the derived value, masking all values $<1$. The $\mathrm{SE}$ images have a $\mathrm{S}: \mathrm{N}$ of $\sim 2$ whereas the $\mathrm{X}$-ray images have values $\sim 0.01$. Application of a simple 'smoothing' filter raises the $\mathrm{S}: \mathrm{N}$ of the $\mathrm{x}$ ray maps to $\sim 2$, a similar value to the SE images. The difference in S:N of $\sim \mathrm{x} 200$ corresponds to the fact that the SE data is 8-bit scaled down from a high collected electron count rate of up to $50 \mathrm{kcps}$, based on approximated detector DQE and Si yield values. The data suggests that with the larger surface area SDD now available (e.g. $\left.80 \mathrm{~mm}^{2}\right)$ then $\mathrm{x}$-ray maps of comparable S:N to SE images and similar pixel size can be collected in $<10 \mathrm{hrs}$. The new WDS with SDD, using high primary beam currents on suitable samples, can achieve counts to $15 \times 10^{6} \mathrm{cps}^{5}$ and so a collection time of $\sim 20$ mins. These data support previously expressed views ${ }^{1}$ that SDD will provide acceptable $\mathrm{S}: \mathrm{N}$ imagery in practical collection times in the near future.

The SE images yield resolution limits around 0.1um for the range of beam energies used, despite the high beam currents and long working distances, re-emphasizing the benefits of a field emission source for x-ray microanalysis. Resolution limit analysis of these initial x-ray maps however is not particularly meaningful other than showing very high eccentricity values that may represent sample or area drift during the accumulation of the x-ray maps.

Data from $80 \mathrm{~mm}^{2}$ SDD and WDS+SDD will be presented against this data set to further extend this investigation.

\section{References}

(1) Newbury, D.E., (2006) Microsc Microanal, 12 (S2:Proc.), 1380 CD.

(2) Griffin, B.J., Joy, D.C. and Michael, J. (2010) Microsc Microanal , 16 (S2:Proc.), 598 CD.

(3) Griffin, B.J., (1979) University of Tasmania Geology Dept, 343, pp. 44

(4) Statham, P., (2006) Microchimica Acta, 155, 289-94.

(5) Lesher, D. (2011) Advanced Microbeam, Pers.comm.

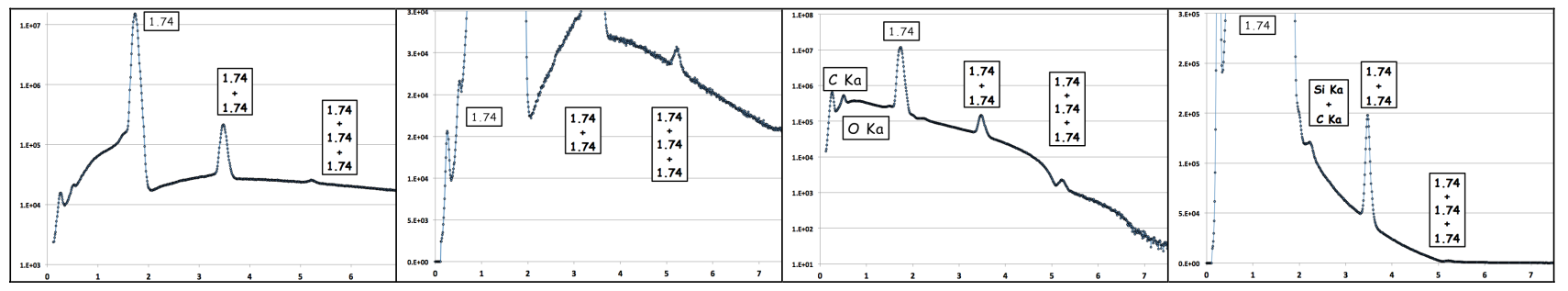

Figure 1: Composite $x$-ray spectra of Si wafer collected using a 30 $\mathrm{mm}^{2}$ SDD on a JEOL 8530F microprobe. From left: $30 \mathrm{kV}-\log$ scale, $30 \mathrm{kV}$ expanded linear scale, $5 \mathrm{kV}-\log$ scale and $5 \mathrm{kV}$ expanded linear scale. The detail of the pile-up peaks is evident in the expanded scale plots. 